\title{
The Design of Quadcopter Frame Based On Finite Element Analysis
}

\author{
Pan Wei ${ }^{1, a}$, ZiJian Yang ${ }^{2, b^{*}}$, Quanzi Wang ${ }^{1, c}$ \\ ${ }^{1}$ Jiangsu Key Laboratory of Large Engineering Equipment Detection and Control, Xuzhou Institute \\ of Technology, Xuzhou, Jiangsu Province, 221000,China \\ 2 Jiangsu Key Laboratory of Large Engineering Equipment Detection and Control, Xuzhou Institute \\ of Technology, Xuzhou, Jiangsu Province, 221000,China \\ ${ }^{3}$ Jiangsu Key Laboratory of Large Engineering Equipment Detection and Control, Xuzhou Institute \\ of Technology, Xuzhou, Jiangsu Province, 221000,China
}

Keywords: Quadcopter Ansys Static and model analysis

Abstract: The frame is an important basis for aircraft structural the design improvements and innovation as a whole frame bearing member. Early in the design stage, a large number of samples are used in the collision and reliability test, which not only causes a waste of resources, but also puts a lot of manpower and resources. So the development cycle is quite lengthy. With the continuing development of computer technology and scientific calculations, the introduction of the finite element method greatly shorten the development cycle frame. More and more machinery and aerospace companies have seen finite element structural analysis as a necessary part of the mechanical development. Using the finite element method, optimization of key parts of the machine and the machine has become an indispensable part of the development process. This paper will analyze the static and modal frame, in order to reflect the important role of finite element analysis in the frame design.

\section{The Introduction and classification of the frame}

Frame is an important component of Quadcopter. It is under enormous pressure, instant impact and partially reversed torque during the flight. In addition, the electronic control system, the motor system are mounted on the frame. Therefore, four aircraft frame must have sufficient strength and rigidity. Based on perfect function of $\mathrm{CAE}$ analysis software and advanced computer hardware, finite element analyses the force of structures reasonably, identify and modify the various components' flaws before specific design is applied. This can avoid significant economic losses due to product failure.

According quadcopter shape, the frame of the aircraft can be divided into two kinds that are profiled frame and X frame. Profiled frame has a larger space and the center of gravity is simple and quick to position. It can support the machine arm and tripod, you can have more expansion holes to facilitate post product upgrades. It takes up little space when folded and is easy to carry. It can be reserved for ultrasonic module mounting holes. X frame's design is simple, good symmetry, more flexible and suitable for stunt flying. But it's aside space is limited and inconvenient for post-upgrade. In this paper, $\mathrm{X}$ frame will be analyzed by finite element as a reference.

\section{Select the shape of section}

When the frame is under bending and twisting load, the amount of deformation is closely related to the cross-sectional shape. First, the stiffness of the solid structure is smaller than the hollow 
structure. Second, the torsional stiffness of a closed circular cross-section is not good, but bending and torsional stiffness of closed square section is good. Third, changing the cross-sectional profile dimensions and wall thickness is the most effective way to change the stiffness. Therefore, this design uses a hollow tube connected the chassis to motor, which can also reduce the overall weight.

\section{Static analysis for frame}

Figure 1, a circular plate has a thickness of $4 \mathrm{~mm}$. On the edge of the plates, the six mounting holes are fixed. The upper plate is under a pressure of $4 \mathrm{e} 3 \mathrm{~Pa}$. We performer static analysis and nonlinear analysis for this plate. The material of the plate is carbon fiber and the material properties are: elastic modulus $\mathrm{E}=2.3 \mathrm{E} 11 \mathrm{~Pa}$, Poisson's ratio $\mu=0.22$.

First, we can build the model and select units. In Pro / e, we can establish reasonable and simplified three-dimensional model and import it into the ANSYS software, then the associated restoration is for its surface and line, and last we select unit as Solid92. Second, we mesh model. Division of unit applies Mesh Tool function of finite element analysis software. As we select unit Solid92, so the density of meshing does not affect the accuracy of the solution. In order to save time, we divide the grid relatively less as well. We choose "Smart Size" 6 for automatic division. Third, we handle the boundary condition. It enables the model to simulate the installation and fixation of structure, the interaction between surrounding structures, and the stress state of structure accurately under the condition of all kinds of work. Fourth, we restrain the boundary conditions. When we calculate the upper plate model, we make $U X=U Z=0$ in the section and take a node $U Y=0$ in the vertical direction. Such models can simulate the real situation of the plate. Fifth, we apply a load to the border. Since the upper plate is squeezed by the electronic device, we add a pressure to the plate, $P_{2}=F_{2} / S_{2}$. ( $F_{2}$-- the maximum rated pressure suffered by this frame is $120 \mathrm{~N} ; S_{2}$--the force area on the upper surface of the moving beam, $S_{2}=1.14 \times 10^{5} \mathrm{~mm}^{2}$ )

Finally, we calculate with the "Solution" of finite element analysis software. The stress contours After solving is showed in Figure 1. The maximum strain on the circular plate is located at the four quadrants. The deformation is $0.0145 \mathrm{~mm}$ and the degree of deformation is $1.45 \%$, higher than the deformation limit $1 \%$ of what the carbon fiber can withstand. Therefore, the structure does not meet the requirements and we need to improve this structure.

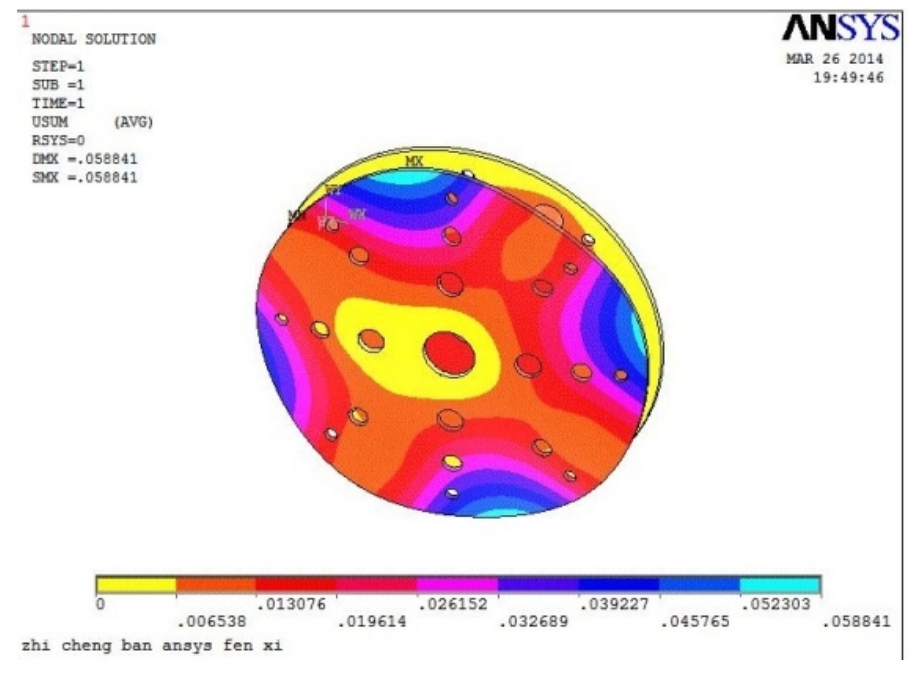

Figure 1 


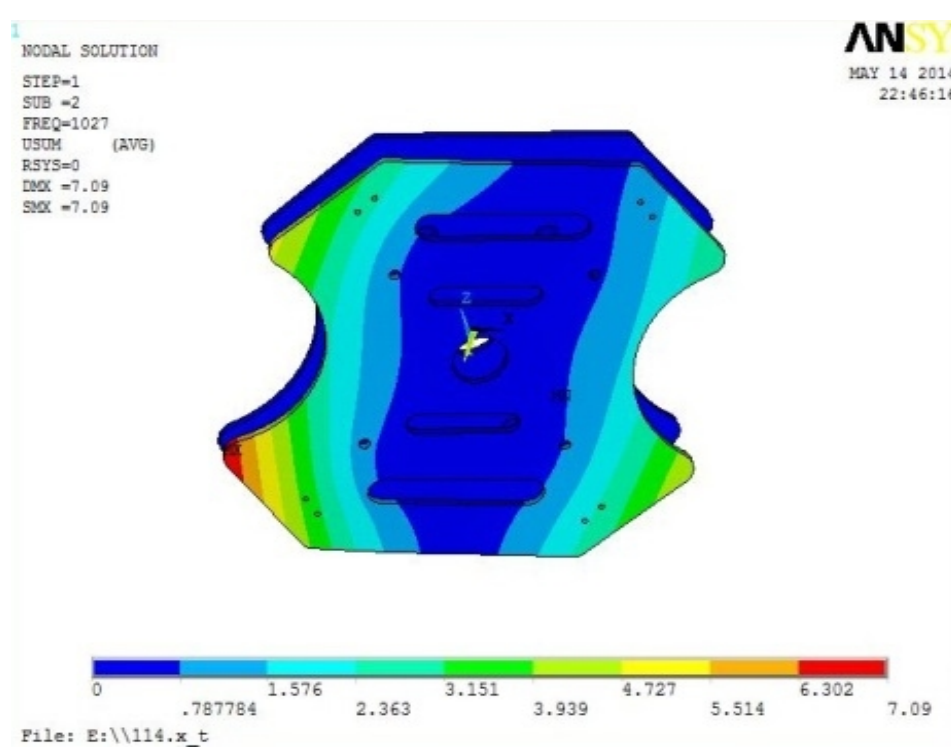

Figure 2

\section{Modal shape analysis for frame}

The material of the frame is carbon fiber and the material properties are: elastic modulus $\mathrm{E}=2.3 \mathrm{e} 11 \mathrm{pa}$, Poisson's ratio $\mu=0.3$, density $\rho=1.5 \mathrm{e} 3 \mathrm{~kg} / \mathrm{m}^{3}$.Figure2. Perform modal analysis for the frame. ANSYS offers seven kinds of modal extraction methods and we use BLOCK LANCZOS method.

First, establish the model. We simplify the model of frame, by removing the auxiliary hole and pattern used for beautification on the frame, because these structures have little effect on the overall performance of the frame. Second, mesh the model. Select "Smart Size" 6 to mesh the model elaborately. Third, perform modal analysis for the model that has been meshed. Impose constraints on the four mounting holes of the shaft and select the type of analysis for "Modal". We proceed to do modal analysis with five bands and choose Block Lanczos Method as analysis mode. We select all items and every step as Output Control option. We also choose five meta-analysis in the extended modal analysis.

Finally, according to its own natural frequency and modal analysis of the frame, we can see that the amplitude is larger on the edge of the frame and the frame is not sensitive to low-frequency vibration. Therefore, quadrocopter should be taken to avoid disturbance of high frequency vibration during descent. In this regard, we propose the following two responses. (1) Reduce the rate of loading and unloading equipment and make loading and unloading is completed in the quasi-static state. (2) The frame can be equipped with vibration isolation equipment that has better high-frequency filtering performance.

\section{Design summary}

This design uses pro / e software for the overall chasse's modeling design, and completes the two-dimensional assembly drawing and component's design, combined with AutoCAD. It improves the traditional design process, efficiency and save manufacturing costs. In order to meet the design requirements, we must consider the following requirements and all aspects, based on the different conditions and environments of using frame. 
First, analyze frame's forms of work in the specific work environment, and establish three-dimensional model of the frame by means of three-dimensional modeling software ProE。 Second, choose the right materials and reduce costs, the weight of the frame. Third, using the finite element software, process geometry model and mesh reasonably to complete finite element model. Fourth, lead the finite element model into ANSYS to calculate and analyze the strength and stiffness of the frame. Fifth, read the results and improve part of the structure, based on the results of static analysis so that we can improve efficiency and structural strength and stiffness of the material.

By analyzing the frame of quadrocopter with the 3D finite element, we can get all kinds of detailed stress and strain contours, modal analysis formation diagrams and corresponding data to guide the improvement of design and decrease of the costs of design, design and analysis cycle time and the consumption of raw materials and capital, which is very convenient and direct.

\section{References:}

[1] Zhao Wuyun, Wu Jingfeng, zhengfengwei, Three farm vehicles, static and dynamic characteristics analysis frame [J], Agricultural Machinery, 2005, 36(11).

[2] Yue Jilong, Zhang Qingjie, zhuhuayong, Advances in micro mini four-rotor UAV and key technical analysis [J], Electro-optical and Control. 2010-10..

[3] Lijun Feng, Zhang Xiong, Theoretical Mechanics [M], Second edition. Beijing Tsinghua University Press.2010:213-216.

[4] Yangguang.2CZ. Mechanical analysis of 21 type sugarcane machine frame and optimization [J]. Agricultural Machinery, 2007, 38(1).

[5] Jes Fenger. Urban air quality. Atmospheric Environment, 1 999, 33: 4877 4900.

[6] Pesters B A V H V. PolyMAX modal parameter estimation from operational data[J]. Leuven: Katholieke University, 2004.

[7] Liujie, Quadrocopter Research and Design, Nanjing University of Posts and master's degree thesis, in April 2013. 